\title{
BMJ Open Timing, delays and pathways to diagnosis of endometriosis: a scoping review protocol
}

\author{
Martha Grace Cromeens (D) , ${ }^{1}$ Erin T. Carey, ${ }^{2}$ Whitney R. Robinson, ${ }^{3,4}$ \\ Kathleen Knafl, ${ }^{1}$ Suzanne Thoyre ${ }^{1}$
}

To cite: Cromeens MG, Carey ET, Robinson WR, et al. Timing, delays and pathways to diagnosis of endometriosis: a scoping review protocol. BMJ Open 2021;11:e049390. doi:10.1136/ bmjopen-2021-049390

- Prepublication history for this paper is available online. To view these files, please visit the journal online (http://dx.doi. org/10.1136/bmjopen-2021049390).

Received 31 January 2021 Accepted 08 June 2021

Check for updates

(C) Author(s) (or their employer(s)) 2021. Re-use permitted under CC BY-NC. No commercial re-use. See rights and permissions. Published by BMJ.

${ }^{1}$ School of Nursing, University of North Carolina at Chapel Hill, Chapel Hill, North Carolina, USA ${ }^{2}$ Department of Obstetrics and Gynecology, University of North Carolina at Chapel Hill School of Medicine, Chapel Hill, North Carolina, USA

${ }^{3}$ Department of Epidemiology, University of North Carolina at Chapel Hill Gillings School of Global Public Health, Chapel Hill, North Carolina, USA

${ }^{4}$ University of North Carolina at Chapel Hill Carolina Population Center, Chapel Hill, North Carolina, USA

Correspondence to Martha Grace Cromeens; cromeens@email.unc.edu

\section{ABSTRACT}

Introduction Pathways to diagnosis for women with endometriosis are frequently characterised with delays. Internationally, women face significant barriers and times to diagnosis. The prolonged time without a diagnosis may result in treatment delay, with clinical implications of chronic pain and an unknown effect on fertility outcomes. As delays in diagnosis extend, those suffering from endometriosis incur more cost and frequently experience a reduction in quality of life. The scoping review described in this protocol will (1) map current international scientific peer-reviewed and grey literature investigating pathways, timing, and delay of diagnosis of endometriosis, (2) define common concepts used in the literature, and (3) identify gaps for future examination and intervention development. Methods and analysis This protocol outlines a scoping review to investigate the current research focused on pathways, timing, and delays in endometriosis diagnosis. The scoping review uses the Joanna Briggs Institute Methodology. The researchers applied the Population, Concept, Context approach to form the research questions. A search string of key terms and Medical Subject Headings will be used to systematically search the PubMed, CINAHL, EMBASE, Web of Science, and Cochrane databases. We will also search ClinicalTrials.gov and grey literature sources. The original search was performed in July 2020 , and it will be rerun prior to the manuscript submission. Finally, the reference lists of included works will be reviewed for additional studies. The search results will be screened and reviewed according to predetermined inclusion and exclusion criteria. Data will be extracted from the studies identified for final inclusion using a predetermined tool. The resulting data will be analysed to report the state of the science.

Ethics and dissemination The proposed scoping review does not require review or approval by an ethical board. The researchers will disseminate the study results via conference presentations and publication in a peerreviewed journal.

\section{INTRODUCTION}

Endometriosis, a gynaecological condition characterised by endometrial stroma and gland like lesions outside of the uterus, has variable clinical presentations. ${ }^{1-3}$ The lesions themselves may be limited to superficial implants, cysts inside the ovary, deep

\section{Strengths and limitations of this study}

This protocol proposes a systematic scoping review of current literature and research on the pathways, timing, and delays of the diagnosis of endometriosis.

- The Joanna Briggs Institute Methodology and recommended Population, Concept, Context method for research question formation is the scaffold on which this protocol builds a systematic approach.

- This review excludes non-English language studies.

- The proposed scoping review offers an effective and comprehensive means to identify gaps for future research into pathways, timing, and delays of diagnosis of endometriosis.

infiltrating disease into the surrounding tissue or pelvic organs or a combination. Similarly, clinical symptoms are also varied, ranging from women who experience no pain, to those with dysmenorrhoea, dyspareunia, dyschezia, or those with a complex pelvic pain presentation with multiple pain complaints. ${ }^{4}$ Although healthcare providers make provisional diagnoses based on symptoms, physical examinations, imaging and treatment response, surgical evaluation with adjunct histological review remains the gold standard of diagnosis. ${ }^{15}$ The requirement of a surgical diagnosis has been challenged for its limitations and risks. ${ }^{6}$ This standard acts as a barrier, compounding obstacles to diagnosis for patients, providers, and health systems. As a result, adults and adolescents with endometriosis internationally experience delays and extended times to diagnosis.

Diagnostic delay may have potentially harmful effects on the central nervous system, as people without a diagnosis may experience more pain over time. Chronic, untreated pain from any cause, including endometriosis, may contribute to dysregulation of the peripheral and central nervous system. ${ }^{7}$ Over time, alterations in pain processing, increases 
the risk of developing abnormal pain referral patterns and may result in a chronic pain presentation. ${ }^{7-11}$

Likewise, the impact of diagnostic delay and infertility should not be overlooked. Endometriosis decreases fertility through several mechanisms including structural abnormalities in reproductive organs, immunological and endocrine dysfunction affecting embryo implantation, and baseline ovarian reserve. ${ }^{12}$ Compared with medical treatment of endometriosis-associated infertility, surgical interventions may have a greater effect on fertility outcomes. $^{13}$

In parallel, individuals with endometriosis incur significant costs in the form of lost work productivity and healthcare expenses. Prolonging the search for a diagnosis and delaying treatment may mean extended times of compounding financial losses. Multiple studies have found that symptomatic individuals report lost work productivity (absenteeism and presenteeism), being out of work up to 10 or more hours per week. ${ }^{14-16}$ Further, people with endometriosis incur significant costs, both direct (eg, emergency department visits, hospitalisations, surgeries and treatments) ${ }^{17-19}$ and indirect (eg, work absenteeism, short-term and long-term disability). ${ }^{14}$ 16-19 Soliman et al found that patients with endometriosis had significantly higher healthcare utilisation and higher annual all-cause expenditures prediagnosis and postdiagnosis compared with control patients. ${ }^{18}$ These financial burdens likely mount as they seek a diagnosis.

Despite what is known about the physical and financial burdens of endometriosis, the systematic reviews on timing, delay, and influencing factors on pathways to diagnosis are limited. In 2013, Culley et al published a critical narrative review of studies reporting the psychological impact of endometriosis. ${ }^{20}$ The 21 studies, categorised by patient-related factors and medical profession-related factors, found diagnostic delay to be a key theme. ${ }^{20}$ This review, while helpful, only captured studies focused on psychological impacts of endometriosis. Two years later, a systematic review of qualitative research on women's experiences with endometriosis was published. ${ }^{21}$ Again, diagnostic delay was a major theme in 10 of the 18 studies reviewed. ${ }^{21}$ While the review outlined major findings concerning delays, it did not elaborate on the concept of diagnosis delay. Furthermore, this review solely considered qualitative studies, limiting the overall research landscape of diagnosis delay data. ${ }^{21}$ In 2017, Soliman et al conducted a quantitative study analysing factors associated with the time to diagnosis including a supplemental table of 16 studies reporting diagnostic delays. ${ }^{22}$ No further analysis of the concepts or literature were presented. In April 2020, a systematic review of diagnostic delay for women with endometriosis using quantitative data to review duration of diagnostic delay was registered with PROSPERO. ${ }^{23}$ To the knowledge of the authors of this protocol, the registered review has not been published. These studies highlight a growing interest regarding factors associated with diagnostic delay of endometriosis.
While these resources represent the reviews and summaries of studies on timing, delay or pathways to diagnosis of endometriosis, there is no systematic scoping review of the qualitative and quantitative available literature on these topics. A systematic scoping review is well suited for the broad objectives of this study, designed to map concepts and identify knowledge gaps. ${ }^{24} \mathrm{~A}$ scoping review methodology is ideal in searching across research designs (eg, quantitative, qualitative, mixed-methods) to determine the range of evidence in a single frame. ${ }^{24}$ Scoping reviews do not analyse nor compare data to make recommendations for clinical practice. ${ }^{24}{ }^{25}$ Instead, scoping reviews map the existing broad scientific research to identify gaps in research and create recommendations for future investigation. ${ }^{24}$

The information assembled from the scoping review will support the development of a uniform language and identify necessary directions for future endometriosis research and interventions. This protocol proposes a systematic scoping review to map the state of the international scientific research on pathways, timing, and delays of diagnosis of endometriosis to identify gaps for future investigation across methodologies.

\section{Rationale}

Delays in diagnosis for women with endometriosis may result in compounding financial, emotional and physical burdens for the women and their communities. Although a recognised problem internationally, there have been no scoping reviews detailing how this phenomenon has been studied. A scoping review of the pathways, timing, and delays in diagnosis of endometriosis will map the existing research, define key concepts, and identify gaps for future research.

\section{OBJECTIVES}

The authors describe a protocol for a systematic scoping review with the primary objective to map current international scientific peer-reviewed and grey literature investigating pathways, timing, and delay of diagnosis of endometriosis. The results of this review will guide recommendations for future research.

\section{METHODS AND ANALYSIS}

This review will follow the Joanna Briggs Institute (JBI) guidelines for scoping reviews. ${ }^{24}{ }^{25}$ Accordingly, an a priori scoping review protocol was developed prior to execution. ${ }^{24}$ Results of the scoping review will be reported consistent with the Scoping Review Extension of the Preferred Reporting Items for Systematic Reviews and Meta-Analyses (PRISMA-ScR) checklist. ${ }^{26}$ An overview of the procedure discussed in the protocol is presented in figure 1 .

\section{Patient and public involvement}

This scoping review will not require patient or public involvement. Patients and the public were not involved 


\begin{tabular}{|c|c|c|c|c|c|}
\hline $\begin{array}{l}\text { Question } \\
\text { Formation }\end{array}$ & $\begin{array}{l}\text { Search Strategy } \\
\text { Development }\end{array}$ & Search & $\begin{array}{c}\text { Screening \& } \\
\text { Full-text Review }\end{array}$ & Data Extraction & $\begin{array}{c}\text { Data Analysis \& } \\
\text { Synthesis }\end{array}$ \\
\hline $\begin{array}{l}\text { PCC Framework } \\
\text { - Population: People } \\
\text { with endometriosis } \\
\text { - Concept: } \\
\text { Pathways/timing/ } \\
\text { delays in diagnosis } \\
\text { - Context: International, } \\
\text { English language }\end{array}$ & $\begin{array}{l}\text { Iterative Process } \\
\text { - Consulted research } \\
\text { librarian } \\
\text { - Research team } \\
\text { consensus } \\
\text { - Finalized with } \\
\text { research librarian }\end{array}$ & $\begin{array}{l}\text { - Databases: PubMed, } \\
\text { CINAHL, EMBASE, } \\
\text { Web of Science, } \\
\text { Cochrane, \& } \\
\text { ClinicalTrials.gov } \\
\text { - Initial search: July } \\
2020 \\
\text { - Search update: } \\
\text { September } 2021\end{array}$ & $\begin{array}{l}\text { - Inclusion/exclusion } \\
\text { criteria } \\
\text { - Two independent } \\
\text { reviewers } \\
\text { - Conflict resolution }\end{array}$ & $\begin{array}{l}\text { - Design extraction tool } \\
\text { with team consensus } \\
\text { - Two independent } \\
\text { extractors } \\
\text { - Conflict resolution }\end{array}$ & $\begin{array}{l}\text { - Reporting consistent } \\
\text { with PRISMA-ScR } \\
\text { - Flow diagram } \\
\text { - Narrative description } \\
\text { - Results of PCC } \\
\text { categories }\end{array}$ \\
\hline
\end{tabular}

Figure 1 Scoping review procedure. PCC, Population-Concept-Context; PRISMA-ScR, Scoping Review Extension of the Preferred Reporting Items for Systematic Reviews and Meta-Analyses.

in the design, conduct, reporting, or dissemination plans of this protocol.

\section{Scoping review questions}

The research questions for this scoping review were formed by applying the Population-Concept-Context (PCC) framework (see table 1) to achieve the primary objective described earlier. ${ }^{24}$

This led to the primary question: What research has been performed internationally (context) concerning the pathways, timing, and delays in diagnosis of endometriosis (concept) for people across all age groups (population)? Secondary review questions were identified to further understanding of the defined PCC and answer the primary question. The primary and secondary review questions can be seen in figure 2 .

\section{Search strategy process}

The search strategy will use the three-step process recommended by JBI. ${ }^{24}$ First, we compiled a list of potential search terms by reviewing titles, abstracts and index terms of key articles found in PubMed and CINAHL. The information gained from the initial search was used to develop a more comprehensive search strategy based on the PCC framework. The list of root terms and the PubMed search string can be seen in table 2. Variants of the terms identified in table 2 were refined to create a final search strategy with search phrases and Medical Subject Headings (MeSH) terms. A research librarian was consulted in the development of the final search strategy. The term 'pathway' and its variants were excluded from the search string because they drew results focused on genetic testing. However, studies focused on pathways to diagnosis in relationship to timing or delay were captured using the terms in table 2. Grey literature such as dissertations and white papers were not filtered out of the search string. The search strategy would capture these materials in the respective databases. Second, the search strategy was reviewed by the team members and then translated for each database being searched. The finalised search strings were used to search six databases (see later). Third, the reference lists of the included articles (determined in the screening process discussed later) were searched for additional studies with the key terms in mind.

\section{Information sources}

Five databases-PubMed, CINAHL, EMBASE, Web of Science and Cochrane-were searched on 1 July 2020. The search did not apply date limits, but excluded non-English language articles. We also searched ClinicalTrials.gov for registered clinical studies focused on pathways, timing, or delays in diagnosis of endometriosis. The search will be repeated prior to the submission of the scoping review results for publication (expected in September 2021).

\section{Eligibility criteria}

The eligibility criteria were built on the PCC framework to answer the research questions. The inclusion and exclusion criteria are outlined in table 3 .

\section{Abstract and full-text screening}

The literature search from all included databases will be uploaded to Endnote, where duplicates will be removed. The remaining results will be imported into Covidence ${ }^{27}$ for the screening process. Covidence also removes duplicates. For the initial screening, two researchers will independently review the titles and abstracts for inclusion.

Table 1 Population-Concept-Context

\begin{tabular}{|c|c|}
\hline Population & All peer-reviewed and grey literature including people with endometriosis across all age groups. \\
\hline Concepts & $\begin{array}{l}\text { Literature reporting research on pathways, timing, or delay in diagnosis of endometriosis will be included in } \\
\text { the review. The concept of 'pathways' includes research on influencing factors leading to a diagnosis such } \\
\text { as the first provider consulted, specialty of providers, numbers of providers and numbers of emergency room } \\
\text { visits as they relate to timing/delay of diagnosis. }\end{array}$ \\
\hline Context & $\begin{array}{l}\text { The context is international. The location, time frame and environment will not be limited. The language of the } \\
\text { articles was limited to English. }\end{array}$ \\
\hline
\end{tabular}




\section{Primary Review Question}

What research has been performed internationally (context) concerning the pathways, timing, and delays in diagnosis of endometriosis (concept) for people across all age groups (population)?

\section{Secondary Review Questions}

- What approaches have researchers utilized to investigate pathways, timing, and delays in diagnosis?

- How have pathways, timing, and delays in diagnosis been defined in research?

- How have the concepts of pathways, timing, and delays in diagnosis been measured?

- What are the characteristics of the samples studied in this research?

- What factors were investigated or identified in relationship to pathways, timing, and delays in diagnosis?

- What impacts of delays in diagnosis were identified or investigated?

Figure 2 Scoping review research questions.

The two screeners will perform the initial screening for $10 \%$ of the records by applying the inclusion and exclusion criteria. Following the screening, they will review conflicting decisions to develop a common understanding of the inclusion/exclusion criteria and improve agreement. Revisions may be made to the inclusion and exclusion criteria through this process. After reviewing $50 \%$ of the cases, a second meeting will be scheduled to resolve conflicts, then proceed with the remaining records. Finally, a third researcher will independently 'tiebreak' any unresolved conflicts.

After completion of the record review, the same two screeners will perform the full-text screening of the

Table 2 Search terms

$\begin{array}{ll}\text { Search terms } & \text { MeSH terms } \\ - \text { Diagnosis } & \text { Delayed diagnosis } \\ \text { Delayed diagnosis } & \text { Time factors } \\ - \text { Late diagnosis } & \\ - \text { Time } & \\ - \text { Delay } & \\ - \text { Endometriosis } & \end{array}$

\section{PubMed search String}

(((“Delayed Diagnosis”[Mesh] OR “Delayed Diagnosis”[tiab] OR "Delayed Diagnoses"[tiab] OR "Late Diagnosis"[tiab] OR "late diagnoses"[tiab] OR ((diagnosis[sh] OR diagnosis[tiab] OR diagnosed[tiab] OR diagnosing[tiab] OR diagnoses[tiab]) AND ("time factors"[mesh] OR delay[tiab] OR delayed[tiab] OR delays[tiab] OR delaying[tiab]))))) AND ((endometriosis[mesh] OR endometriosis[tiab] OR Endometrioses[tiab])) Filters: English records that passed the initial abstract screening. Again, the two screeners will review $10 \%$ of the articles for inclusion, and then meet to resolve conflicts and refine their application of the inclusion/exclusion criteria. In cases of record disagreement, a third researcher will resolve conflicts. The same process will repeat for the first $50 \%$ of the records, and again for the final $50 \%$ of the cases. The cases that make it through the full-text review will comprise the sample for data extraction and references from included articles will be reviewed to identify additional potential articles and grey literature (eg, dissertations, white papers). If any materials are chosen from the reference lists, they will be added to Covidence and they will undergo the process of abstract and full-text screening.

\section{Data extraction and charting}

A preliminary data extraction tool was created based on the objective, PCC framework, and resulting research questions. Four researchers will perform an initial review of articles. As a group, they will edit and refine the initial extraction tool to better meet the study objective and review questions. ${ }^{25}$ The data extraction fields will be chosen to further explore population, concept, and context. Possible data extraction categories for each can be seen in table 4 . The final sample of articles will be divided equally among the reviewers, with two researchers reviewing each article: the primary author and an additional reviewer. The extraction tool will be used to collect and chart data. The two reviewers will meet to resolve conflicts, hone their shared understanding of the extraction method and refine the extraction tool when needed. A third researcher will resolve unreconciled conflicts. 
Table 3 Inclusion and exclusion criteria

\begin{tabular}{ll} 
Inclusion criteria & Exclusion criteria \\
\hline Studies with participants of any age, race/ethnicity, & Animal studies. \\
nationality or socioeconomic status. & Abstracts only. \\
Study participants identified as having endometriosis (ie, & Editorials, Op-eds or position papers. \\
surgical verification, histological confirmation, provider & Literature reviews. \\
presumed or participant identified). & Case reports. \\
The purpose and/or results of the study report issues & Pathways, timing, or delays in diagnosis are reported solely \\
related to pathways, timing and/or delay in diagnosis. & as a descriptive statistic of the sample. \\
Primary research/empirical studies (qualitative, quantitative, & Full-text is not published in English. \\
mixed-methods and intervention studies). & Studies concentrating on diagnostic tools, tests or \\
Peer-reviewed journals. & equipment. \\
Grey sources (eg, dissertations). & Studies in which the participants are solely healthcare \\
Case studies. & providers and focus on knowledge base, understanding and \\
& opinions concerning endometriosis.
\end{tabular}

\section{Data analysis and synthesis of results}

The results of the search strategy and the screening process will be reported consistent with the PRISMA-ScR recommended method with a flow diagram and corresponding narrative description. ${ }^{26}$ The researchers will report the synthesis of the data from the extraction tool for the PCC categories (table 4). The results will be reported to answer the primary objective and research questions (figure 2).

\section{DISCUSSION (ETHICS AND DISSEMINATION)}

Approval from the research ethics boards of the University of North Carolina at Chapel Hill is not required for this scoping review. The scoping review did not require patient or public involvement, limiting ethical and safety considerations. The results of this scoping review will be disseminated through academic, clinical, and public venues. The researchers will seek publication for the results in peer-reviewed journals, and present the review findings at conferences. The researchers also intend to form recommendations for areas of future research.

One limitation of this review is the language restriction. The researchers limited inclusion to articles published in English. Articles that had an English abstract, but nonEnglish body were excluded. Endometriosis and delays in diagnosis of endometriosis are challenges experienced and researched globally. The science would benefit from a scoping review inclusive of other languages.
The common phenomenon of prolonged average times to diagnosis represents one of the most challenging aspects of endometriosis for the patients and their healthcare providers. The scoping review of delay, timing, and pathways to diagnosis described in this protocol will survey the current scientific literature to identify gaps in the research across methods to encourage uniformity of terms and prevent duplication of efforts. International consensus on definitions and concepts while recognising past research approaches to understand influencing factors, relationships, and impacts of delays in diagnosis of endometriosis will lead to more efficient research, targeted interventions, and ultimately improved outcomes for patients.

\section{Twitter Martha Grace Cromeens @mgcromeens}

Contributors All five authors contributed to the design, search strategy and writing of the scoping review protocol. MGC, ETC, WRR, KK, SMT made substantial contributions to conception and design, or acquisition of data, or analysis and interpretation of data. MGC, ETC, WRR, KK, SMT involved in drafting the manuscript or revising it critically for important intellectual content. MGC, ETC, WRR, KK, SMT given final approval of the version to be published. Each author should have participated sufficiently in the work to take public responsibility for appropriate portions of the content. MGC, ETC, WRR, KK, SMT agreed to be accountable for all aspects of the work in ensuring that questions related to the accuracy or integrity of any part of the work are appropriately investigated and resolved.

Funding This research is supported by the National Institute of Nursing Research of the National Institutes of Health under award number F31NR018786 (Cromeens, PI), a UNC Dissertation Completion Fellowship and a 2018 Sigma Small Grant by Sigma Theta Tau International (STTI \#19-2191). MGC is also a Virginia Kelley Scholar of the American Nurses Foundation (ANF 10055), a Linda Waring Matthews

Table 4 Extraction categories

\begin{tabular}{lll}
\hline Population & Concept & Context \\
\hline Sample size. & Definition of pathway, timing or delay. & Geographic origin of study. \\
Means of diagnosis of endometriosis. & Means of calculation. & Recruitment setting. \\
Race/ethnicity. & Factors investigated in relationship to & Recruitment means. \\
Socioeconomic status (proxies & pathway, timing or delay. & Data collection setting. \\
reported). & Impacts of delay and diagnosis on & Data collection means. \\
Demographics reported. & patients' lives. & \\
Health descriptors reported. & & \\
\hline
\end{tabular}


Research Scholar, 2019-2020 and a Hillman Scholar in Nursing Innovation at the School of Nursing at the University of North Carolina at Chapel Hill. This study also received funding from the Hillman Scholars Programme in Nursing Innovation through the 'Interdisciplinary Inquiry' and the 'Advancing Early Research Opportunities' grants. WRR's research, 'Racial differences in treatment with hysterectomy: A multilevel investigation', is supported by the National Institute on Minority Health and Health Disparities of the National Institutes of Health under award number R01MD011680 (Robinson, PI).

Competing interests None declared.

Patient and public involvement Patients and/or the public were not involved in the design, or conduct, or reporting, or dissemination plans of this research.

Patient consent for publication Not required.

Provenance and peer review Not commissioned; externally peer reviewed.

Open access This is an open access article distributed in accordance with the Creative Commons Attribution Non Commercial (CC BY-NC 4.0) license, which permits others to distribute, remix, adapt, build upon this work non-commercially, and license their derivative works on different terms, provided the original work is properly cited, appropriate credit is given, any changes made indicated, and the use is non-commercial. See: http://creativecommons.org/licenses/by-nc/4.0/.

ORCID iD

Martha Grace Cromeens http://orcid.org/0000-0002-0551-5172

\section{REFERENCES}

1 Giudice LC, Kao LC. Endometriosis. The Lancet 2004;364:1789-99.

2 Zondervan KT, Becker CM, Missmer SA. Endometriosis. N Engl J Med 2020;382:1244-56.

3 Zondervan KT, Becker CM, Koga K, et al. Endometriosis. Nat Rev Dis Primers 2018;4:9.

4 Vercellini P, Viganò P, Somigliana E, et al. Endometriosis: pathogenesis and treatment. Nat Rev Endocrinol 2014;10:261-75.

5 Kinkel K, Brosens J, Brosens I, et al. Preoperative Investigations. In: Sutton C, Jones K, Adamson GD, eds. Modern management of endometriosis. London, England: Taylor and FrancisGroup, 2006: 71-85.

6 Agarwal SK, Chapron C, Giudice LC, et al. Clinical diagnosis of endometriosis: a call to action. Am J Obstet Gynecol 2019;220:354. e1-354.e12.

7 Stratton P, Berkley KJ. Chronic pelvic pain and endometriosis: translational evidence of the relationship and implications. Hum Reprod Update 2011:17:327-46.

8 Aredo JV, Heyrana KJ, Karp Bl, et al. Relating chronic pelvic pain and endometriosis to signs of sensitization and myofascial pain and dysfunction. Semin Reprod Med 2017;35:088-97.
9 Bajaj P, Bajaj P, Madsen $\mathrm{H}$, et al. Endometriosis is associated with central sensitization: a psychophysical controlled study. J Pain 2003;4:372-80.

10 Vuontisjärvi S, Rossi H-R, Herrala S, et al. The long-term footprint of endometriosis: population-based cohort analysis reveals increased pain symptoms and decreased pain tolerance at age 46 years. $J$ Pain 2018:19:754-63.

11 Morotti M, Vincent K, Becker CM. Mechanisms of pain in endometriosis. Eur J Obstet Gynecol Reprod Biol 2017;209:8-13.

12 Practice Committee of the American Society for Reproductive Medicine. Endometriosis and infertility: a Committee opinion. Fertil Steril 2012;98:591-8.

13 Lee D, Kim SK, Lee JR, et al. Management of endometriosis-related infertility: considerations and treatment options. Clin Exp Reprod Med 2020;47:1-11.

14 Fourquet J, Báez L, Figueroa M, et al. Quantification of the impact of endometriosis symptoms on health-related quality of life and work productivity. Fertil Steril 2011;96:107-12.

15 Nnoaham KE, Hummelshoj L, Webster P, et al. Impact of endometriosis on quality of life and work productivity: a multicenter study across ten countries. Fertil Steril 2011;96:366-73.

16 Soliman AM, Coyne KS, Gries KS, et al. The effect of endometriosis symptoms on absenteeism and Presenteeism in the workplace and at home. J Manag Care Spec Pharm 2017;23:745-54.

17 Gao X, Outley J, Botteman M, et al. Economic burden of endometriosis. Fertil Steril 2006;86:1561-72.

18 Soliman AM, Surrey E, Bonafede M, et al. Real-World evaluation of direct and indirect economic burden among endometriosis patients in the United States. Adv Ther 2018;35:408-23.

19 Soliman AM, Taylor HS, Bonafede M, et al. Incremental direct and indirect cost burden attributed to endometriosis surgeries in the United States. Fertil Steril 2017:107:1181-90.

20 Culley L, Law C, Hudson N, et al. The social and psychological impact of endometriosis on women's lives: a critical narrative review. Hum Reprod Update 2013;19:625-39.

21 Young K, Fisher J, Kirkman M. Women's experiences of endometriosis: a systematic review and synthesis of qualitative research. J Fam Plann Reprod Health Care 2015;41:225-34.

22 Soliman AM, Fuldeore M, Snabes MC. Factors associated with time to endometriosis diagnosis in the United States. J Womens Health 2017;26:788-97.

23 Illum LRH, Rytter D, Forman A. A systematic review of the diagnostic delay for women with endometriosis. In. PROSPERO 2020.

24 Peters MDJ, Godfrey CM, Khalil H, et al. Guidance for conducting systematic scoping reviews. Int J Evid Based Healthc 2015;13:141-6.

25 Peters MDJ, Godfrey CM, Mclnerney P. Chapter 11: Scoping Reviews. In: Aromataris E, Munn Z, eds. JBI Manual for Evidence Synthesis, JBI, 2020, 2020.

26 Tricco AC, Lillie E, Zarin W, et al. PRISMA extension for scoping reviews (PRISMA-ScR): checklist and explanation. Ann Intern Med 2018;169:467-73.

27 Veritas Health Innovation. Covidence systematic review software, 2021. Available: https://www.covidence.org/ 